\title{
ON REAL CONTINUOUS SOLUTIONS OF ALGEBRAIC DIFFERENCE EQUATIONS
}

\author{
S. M. SHAH
}

1. Introduction. This paper considers the behaviour at infinity of real continuous solutions of algebraic difference equations

$$
P(y(x+m), \cdots, y(x), x)=0
$$

where $P$ is a polynomial with real coefficients in its arguments $y(x+m), \cdots, y(x)$ and $x$. The problem was first treated by Lancaster, ${ }^{1}$ who obtained an upper bound for the rate of increase of the solutions of algebraic difference equations of a given order and pointed out the surprising dissimilarity with the known rates of increase for solutions of differential equations of the same order.

The main object of this paper is to show that any real continuous solution of an algebraic difference equation of the first order

$$
P(y(x+1), y(x), x)=0
$$

satisfies the inequality

$$
\liminf _{x \rightarrow \infty} \log \log |y(x)| / x<\infty .
$$

This is an improvement over the results of Lancaster, who proved that a continuous solution $y(x)$ cannot equal or exceed $c e_{2}\left(x l_{n} x\right)$ for all $x>x_{0}$, where $l_{n}(x)$ is the $n$th iterate of $\log x, e_{2}(x)=e^{e^{x}}, n$ is any fixed positive integer, and $c$ is any positive constant. The essential difference in the rates of increase of the solutions of algebraic differential and difference equations is clearly emphasized when this new result is compared with the results of Borel, who showed that any real continuous solution $y(x)$ of an algebraic differential equation of the first order satisfies the inequality

$$
\limsup _{x \rightarrow \infty} \log \log |y(x)| / \log x<\infty .
$$

Simple examples reveal that relation (3) is the best possible result. First this is the best possible limit, for when $y(x)=a^{b^{x}}$, a solution of

\footnotetext{
Received by the editors February 4, 1946, and, in revised form, November 25, 1946.

${ }^{1}$ Otis E. Lancaster, Some results concerning the behavior at infinity of real continuous solutions of algebraic difference equations, Bull. Amer. Math. Soc. vol. 46 (1940) pp. 169-177. References to the papers of Borel, Vijayaraghavan and others will be found in this paper.
} 
$y(x+1)-(y(x))^{b}=0$, we have

$$
\lim _{x \rightarrow \infty} \log \log |y(x)| / x=\log b .
$$

Since $b$ is any rational number, $\log b$ can be made to exceed any finite value. Second, it is not possible to replace limit inferior by limit or limit superior without restrictive hypothesis on $y(x)$, for if $\psi(x)$ is any increasing continuous function tending to infinity with $x$, a solution of

$$
\left(y(x)-x^{b}\right)\left(y(x+1)-(x+1)^{b}\right)=0
$$

can exceed $\psi(x)$ for a sequence of values of $x$ tending to infinity. The difference equation (5) is satisfied by the continuous function

$$
\begin{aligned}
& y(x)=x^{b} \\
& \text { (for } 2 n-1 \leqq x \leqq 2 n) \\
& =(2 n)^{b}+2\left\{\phi(2 n+1 / 2)-(2 n)^{b}\right\}(x-2 n) \\
& \text { (for } 2 n \leqq x \leqq 2 n+1 / 2) \\
& =(2 n+1)^{b}+2\left\{(2 n+1)^{b}-\phi(2 n+1 / 2)\right\}(x-2 n-1) \\
& \text { (for } 2 n+1 / 2 \leqq x \leqq 2 n+1)
\end{aligned}
$$

$(n=1,2,3, \cdots)$ where $\phi$ is an arbitrary function. Let $\phi(x)=e_{3}(\psi(x))$. Then

$$
\lim _{n \rightarrow \infty} \frac{\log \log y(2 n+1 / 2)}{\psi(2 n+1 / 2)}=\lim _{n \rightarrow \infty} \frac{e^{\psi(2 n+1 / 2)}}{\psi(2 n+1 / 2)}=\infty,
$$

which establishes the above statement.

2. Statements of the theorems. In $\S \S 4-6$ we shall prove the following three theorems on the algebraic difference equations of the first order. In $\$ \$ 7-10$ we consider equations of the $m$ th order.

THEOREM 1. If $y(x)$ be a real continuous solution of a first order algebraic difference equation $P=0$, then $|y(x)|$ cannot equal or exceed $e_{2}(A x)$ for all ${ }^{2} x>x_{0}(A), A$ being a positive constant depending on the given equation $P=0$.

In what follows we suppose throughout that $y(x)$ is real and continuous for $x>x_{0}$.

THEOREM 2. If $y(x)$, a solution of an algebraic difference equation of the first order, be such that ${ }^{3}$

${ }^{2} x_{0}$ is not necessarily the same at each occurrence.

${ }^{3}$ The condition (6) can be replaced by a less stringent one, $\log |y(x)| / \log x>A_{1}$ for all $x>x_{0}\left(A_{1}\right)$, where $A_{1}$ is a constant depending on the given equation. 


$$
\log |y(x)| / \log x \rightarrow \infty
$$

then $|y(x)|<e_{2}(A x)$ for all $x>x_{0}(A)$, where $A$ is a positive constant. depending on the given equation.

THEOREM 3. If $y(x)$, a solution of an algebraic difference equation of the first order, be such that

$$
y(x) \text { is monotonic }
$$$$
\text { for } x>x_{0}
$$

then $|y(x)|<e_{2}(A x)$ for all $x>x_{0}(A)$, where $A$ is positive constant depending on the given equation.

3. Lemmas. We require three lemmas.

LEMMA 1. If $f(x)$ is continuous $s^{4}$ and non-negative for $x>x_{0}$ and if

$$
\limsup _{x \rightarrow \infty} \log \log f(x) / x \geqq A>0
$$

then there exists a sequence of numbers $x_{1}, x_{2}, \cdots, x_{n} \rightarrow \infty$ such that

$$
f(x+1) \geqq\{f(x)\}^{B}
$$

for $x=x_{1}, x_{2}, \cdots ; B$ is any positive number less than $\exp (A)$.

For if the lemma is false then for all $x \geqq X_{0}>x_{0}$

$$
f(x+1)<\{f(x)\}^{B}
$$

and so $f\left(X_{0}+p\right)<\left\{f\left(X_{0}\right)\right\}^{B_{p}}$. Hence

$$
\limsup _{p \rightarrow \infty} \log \log f\left(X_{0}+p\right) /\left(X_{0}+p\right) \leqq \log B<A,
$$

which contradicts the hypothesis and so the lemma is proved.

LEMMA 2. If $f(x)$ is continuous and nondecreasing for $x>x_{0}$ and if (8) holds then there exists a sequence of numbers $x_{1}, x_{2}, \cdots, x_{n} \rightarrow \infty$ such that ${ }^{5}$

$$
\begin{aligned}
f(x) & \geqq e_{2}(\phi(x)), \\
f(x+1) & \geqq\{f(x)\}^{B}
\end{aligned}
$$

for $x=x_{1}, x_{2}, x_{3}, \cdots ; B$ is any positive number less than $\exp (A)$ and $\phi(x)$ is any nondecreasing continuous function such that $\lim _{x \rightarrow \infty} \phi(x) / x$ $=0$.

PRoOF. If $f(x) \geqq e_{2}(\phi(x))$ for all large $x$, then the result follows from

4 Continuity of $f(x)$ is utilized in Lemma 2.

- We prove a result more general than the one we require later on. 
Lemma 1. Suppose therefore that $f(x)<e_{2}(\phi(x))$ for a sequence of values of $x$ tending to infinity. Let

$$
F(x)=f(x) / e_{2}(\phi(x))
$$

and $X>x_{0}$ be an arbitrary large number such that $F(X)<1$. Let $\log B<C<A$. Take $Y>X$ such that $F(Y)>1$ and $\log \log f(Y)>C Y$ $+\phi(Y)$. This is possible since $\lim \sup _{x \rightarrow \infty} \log \log f(x) / x \geqq A>C$. Let $Z$ be the largest number such that $X<Z<Y$ and $F(Z)=1$. Since $F(x)$ is continuous for $x \geqq X, Z$ exists, and $F(x) \geqq 1$ for $Z \leqq x \leqq Y$. Consider $f(Z), f(Z+1), \cdots, f(Z+m)$ where $Y \leqq Z+m<Y+1$.

$$
\begin{aligned}
\log \log f(Z+m) & \geqq \log \log f(Y) \\
& >C Y+\phi(Y) \\
& >C(Z+m-1)+\phi(Z) \\
& =C(Z+m-1)+\log \log f(Z) .
\end{aligned}
$$

Hence

$$
\log \log f(Z+m)-\log \log f(Z)>C(Z+m-1)>C m .
$$

Hence at least one of the values

$$
\log \log f(Z+n)-\log \log f(Z+n-1) \quad(n=1,2, \cdots, m)
$$

is greater than $C$. If $\log \log f(Z+N)-\log \log f(Z+N-1)>C$ then

$$
f(Z+N-1) \geqq e_{2}(\phi(Z+N-1))
$$

and

$$
f(Z+N) \geqq\{f(Z+N-1)\}^{\circ}>\{f(Z+N-1)\}^{B}
$$

which proves the lemma.

LEMMA 3. If $f(x)$ is continuous and nondecreasing for $x>x_{0}$ and if (8) holds, then ${ }^{5}$ there exists a sequence of numbers $x_{1}, x_{2}, \cdots, x_{n} \rightarrow \infty$ such that

$$
\begin{aligned}
& f(x+1) \geqq e_{2}(\phi(x)), \\
& f(x+1) \geqq\{f(x)\}^{B}, \\
& f(x+2) \geqq\{f(x+1)\}^{b},
\end{aligned}
$$

for $x=x_{1}, x_{2}, \cdots ; \phi(x)$ is any increasing continuous function such that $\lim _{x \rightarrow \infty} \phi(x) / x=0$, and $0<B<\exp (A / 2)$.

Proof. Let $0<\epsilon<1-(2 \log B / A)$. By Lemma 2 we can choose a sequence $X_{1}, X_{2}, \cdots$ such that 


$$
\begin{gathered}
f(x) \geqq e_{2}(\phi(x)), \\
\log \log f(x+1)-\log \log f(x)>A(1-\epsilon)
\end{gathered}
$$

for $x=X_{1}, X_{2}, \cdots$. Let $\xi_{r}$ be such that $X_{r}<\xi_{r}<1+X_{r}$ and

$$
\log \log f\left(\xi_{r}\right)=(1 / 2)\left(\log \log f\left(1+X_{r}\right)+\log \log f\left(X_{r}\right)\right) .
$$

$\xi_{r}$ exists since $f(x)$ is continuous and nondecreasing. Since $\xi_{r}-1<X_{r}$ $<\xi_{r}<1+X_{r}<1+\xi_{r}$ we have

$\log \log f\left(\xi_{r}\right)-\log \log f\left(\xi_{r}-1\right)$

$$
\geqq \log \log f\left(\xi_{r}\right)-\log \log f\left(X_{r}\right)>A(1-\epsilon) / 2,
$$

$\log \log f\left(1+\xi_{r}\right)-\log \log f\left(\xi_{r}\right)$

$$
\geqq \log \log f\left(1+X_{r}\right)-\log \log f\left(\xi_{r}\right)>A(1-\epsilon) / 2 .
$$

Hence writing $x_{r}$ for $\xi_{r}-1$ and $d=\exp (A(1-\epsilon) / 2)$, we have

$$
f\left(1+x_{r}\right)>\left(f\left(x_{r}\right)\right)^{d}, f\left(2+x_{r}\right)>\left(f\left(1+x_{r}\right)\right)^{d} .
$$

Also

$$
f\left(1+x_{r}\right)=f\left(\xi_{r}\right) \geqq f\left(X_{r}\right) \geqq e_{2}\left(\phi\left(X_{r}\right)\right) \geqq e_{2}\left(\phi\left(\xi_{r}-1\right)\right)=e_{2}\left(\phi\left(x_{r}\right)\right) .
$$

Hence the lemma is proved.

4. Proof of Theorem 1. Let the given equation be (2). Among the terms of the polynomial $P$ there is a term

$$
T^{\prime}=a^{\prime} x^{\alpha^{\prime}} y(x)^{\beta_{0}^{\prime}} y(x+1)^{\beta_{1}^{\prime}}
$$

which has the property that if

$$
T=a x^{\alpha} y(x)^{\beta_{0}} y(x+1)^{\beta_{1}}
$$

is any other term of the polynomial, then in the sequence of differences

$$
\beta_{1}^{\prime}-\beta_{1}, \quad \beta_{0}^{\prime}-\beta_{0}, \quad \alpha^{\prime}-\alpha
$$

the first nonzero term is positive. $T^{\prime}$ is called the principal term. Divide the equation (2) by $T^{\prime}$. Then all terms except one $T^{\prime} / T^{\prime}$ are of the form $T / T^{\prime}$ where

$$
T / T^{\prime}=b x^{\alpha-\alpha^{\prime}}\{y(x)\}^{\beta_{0}-\beta_{0} 0^{\prime}}\{y(x+1)\}^{\beta_{1}-\beta_{1^{\prime}}} .
$$

These ratios $T / T^{\prime}$ are of three possible types

$$
b\left\{\frac{x^{K_{0}} y(x)^{K_{1}}}{y(x+1)}\right\}^{\beta_{1}^{\prime}-\beta_{1}}, \quad \beta_{1}^{\prime}>\beta_{1},
$$




$$
\begin{gathered}
b\left\{\frac{x^{K 2}}{y(x)}\right\}^{\beta_{0}^{\prime}-\beta_{0}}, \\
b x^{\alpha-\alpha^{\prime}},
\end{gathered}
$$

Let $K$ be the $\max \left|K_{1}\right|$ for all the ratios of type (14) and let ${ }^{6}$

$$
A=1+\max (1, \log K) \text {. }
$$

(i) First, assume that $e_{2}(A x)$ is a solution of (2). If we replace $y(x)$ by $e_{2}(A x)$, the limit of these expressions (14), (15) and (16), as $x$ tends to infinity, is zero. Hence we have a contradiction to the assumption that $e_{2}(A x)$ is a solution of (2). For there exists an $x_{0}$ such that for $x>x_{0}$, the sum of all the terms $T / T^{\prime}$ is less than one in absolute value whereas $T^{\prime} / T^{\prime}=1$.

(ii) Second, assume that a solution $y(x)$ is greater than or equal to $e_{2}(A x)$ for all $x>x_{0}$. Then the ratios $T / T^{\prime}$ are again of the three types (14), (15) and (16). The ratios of types (15) and (16) approach zero as $x$ tends to infinity; and the ratios of type (14) are in absolute value less than

$$
\left\{\frac{y(x)^{K+\epsilon}}{y(x+1)}\right\}^{\beta_{1}^{\prime}-\beta_{1}} \quad\left(x>X_{0}(\epsilon)\right)
$$

and the last expression, by Lemma 1 , tends to zero as $x=x_{n} \rightarrow \infty$; for $K+\epsilon<e K / 2=B$ and $e K / 2<e K=e^{1+\log K} \leqq e^{A}$. Hence we have a contradiction to the assumption that $y(x)$ is greater than or equal to $e_{2}(A x)$ for all $x>x_{0}$.

(iii) Third, assume that $y(x)$ is less than or equal to $-e_{2}(A x)$ for all $x>x_{0}$. Let $y(x)=-Y(x)$. The argument in (i) and (ii) applies to the transformed equation and so we get a contradiction to the assumption (iii). Since $y(x)$ is continuous, the theorem follows.

5. Proof of Theorem 2. By hypothesis (6), $y(x)$ is either positive or negative for all $x>x_{0}$.

If $y(x) \geqq e_{2}(A x)$ for a sequence of values of $x$ tending to infinity, $A$ being the constant defined in the proof of Theorem 1 , then by Lemma 1 , we can find a sequence $x_{1}, x_{2}, \cdots, x_{n} \rightarrow \infty$ such that

$$
y(x+1) \geqq\{y(x)\}^{B}
$$

where $B<\exp (A)$, for $x=x_{1}, x_{2}, \cdots$. The ratios of type (16) tend to zero as $x$ tends to infinity and by hypothesis (6), the ratios of type (15) tend to zero as $x$ tends to infinity. Further the ratios of type (14)

${ }^{6}$ It is possible to choose a lower value of $A$. For instance in Theorems $1,2,3$, we may take $A$ to be any number greater than $\max (\log K, 0)$. 
tend to zero as $x=x_{n} \rightarrow \infty$. Hence we have a contradiction and so $y(x)<e_{2}(A x)$ for $x>x_{0}$.

If $y(x) \leqq-e_{2}(A x)$ for a sequence of values of $x$ tending to infinity, then $y(x)$ is negative for $x>x_{0}$ and we take $y(x)=-Y(x)$. The above argument then applies and the theorem follows.

6. Proof of Theorem 3. We may suppose that $y(x)$ is nondecreasing (for otherwise we can transform the equation by taking $y(x)=-Y(x)$ ) for $x>x_{0}$. If $y(x) \geqq e_{2}(A x)$ for a sequence of values of $x$ tending to infinity, $A$ being the constant defined in the proof of Theorem 1, then by Lemma 2 , we can find a sequence $x_{1}, x_{2}, \cdots, x_{n} \rightarrow \infty$ such that

$$
y(x) \geqq \exp (x), \quad y(x+1) \geqq(y(x))^{B},
$$

where $0<B<\exp (A)$, for $x=x_{1}, x_{2}, \cdots$. Hence the ratios of types (14), (15) and (16) tend to zero as $x=x_{n} \rightarrow \infty$. Hence we have a contradiction and so $y(x)<e_{2}(A x)$ for $x>x_{0}$. Since $y(x)$ is nondecreasing

$$
|y(x)|<e_{2}(A x) \quad \text { for } x>x_{0} .
$$

7. Equations of the $m$ th order. We now consider algebraic difference equations

(1) $\quad P(y(x+m), \cdots, y(x), x)=\sum a x^{\alpha} y(x)^{\beta_{0}} \cdots y(x+m)^{\beta_{m}}=0$

of order $m>1$. Let the principal term be

$$
T^{\prime}=a^{\prime} x^{\alpha^{\prime}} y(x)^{\beta_{0}^{\prime}} \cdots y(x+m)^{\beta_{m}^{\prime}}
$$

so that in the sequence of differences

$$
\beta_{m}^{\prime}-\beta_{m}, \beta_{m-1}^{\prime}-\beta_{m-1}, \cdots, \beta_{0}^{\prime}-\beta_{0}, \alpha^{\prime}-\alpha
$$

the first nonzero term is positive.

We can show, as in first part of Theorem 1, that $c e_{2}(A x)$, where $c$ is any finite constant and $A$ a constant depending on $P=0$, cannot be $a$ solution of $P=0$.

THEOREM 4. Let $P=0$ be an algebraic difference equation of the mth order. If the terms of the equation are so related that when

$$
\beta_{m}^{\prime}=\beta_{m} \text { then } \quad \beta_{i}^{\prime} \geqq \beta_{i} \quad(i=0,1,2, \cdots, m-1)
$$

and if $y(x)$ be a solution of the equation then $|y(x)|$ cannot exceed $e_{2}(A x)$ for all $x>x_{0}(A), A$ being a positive constant depending on $P=0$.

THEOREM 5. Let $P=0$ be an algebraic difference equation of the mth order. If the terms of the equation are so related that when $\beta_{m}^{\prime}=\beta_{m}$ then 
either $\beta_{m-1}^{\prime}>\beta_{m-1}$ or $\beta_{m-1}^{\prime}=\beta_{m-1}$ and $\beta_{i}^{\prime} \geqq \beta_{i}(i=0,1,2, \cdots, m-2)$ and if $y(x)$ be a solution satisfying conditions (6) and (7), then

$$
|y(x)|<e_{2}(A x) \quad \text { for all } x>x_{0}(A) \text {, }
$$

$A$ being a positive constant depending on $P=0$.

COROLLARY. If $y(x)$, a solution of an algebraic difference equation of the second order, satisfies the conditions (6) and (7), then

$$
|y(x)|<e_{2}(A x) \quad \text { for all } x>x_{0}(A),
$$

where $A$ is a constant depending on the given equation.

THEOREM 6. If $y(x)$, a solution of an algebraic difference equation of the second order, be monotonic for $x>x_{0}$, then

$$
\liminf _{x \rightarrow \infty} \log \log |y(x)| / x<\infty .
$$

THEOREM 7. If $y(x)$, a solution of an algebraic difference equation of the mth order, be such that

$$
|y(x+1)| \geqq c|y(x)|^{\Lambda_{2}}
$$

where $c$ is any positive constant and $A_{2}$ is a positive constant depending on the given equation, for $x=a, a+1, a+2, \cdots$, then

$$
\lim _{x \rightarrow \infty} \inf \log |y(x)| / \log x<A_{2} \text {. }
$$

8. Proof of Theorem 4. Under the hypothesis, the ratios $T / T^{\prime}$ take the forms

$$
\begin{gathered}
b\left\{\frac{x^{K_{0}} y(x)^{K_{1}} \cdots y(x+m-1)^{K_{m}}}{y(x+m)}\right\}^{\beta_{m}^{\prime}-\beta_{m}} \quad\left(\beta_{m}^{\prime}>\beta_{m}\right), \\
b\left\{\frac{x^{k_{0}}}{y(x)^{k_{1}} \cdots y(x+m-1)^{k_{m}}}\right\}
\end{gathered}
$$

where $k_{1}, k_{2}, \cdots$ are all non-negative constants and $k_{0}$ is negative if all $k_{1}, k_{2}, \cdots, k_{m}$ are zero. Let $M_{i}$ be the maximum value of all $\left|K_{i}\right|$ for the ratios of type (22) and let $\max \left(\left(M_{0}+M_{1}+M_{2}+\cdots\right.\right.$ $\left.\left.+M_{m}\right), e\right)=K$ and $^{6} A=1+\log K$.

If $y(x) \geqq e_{2}(A x)$ for all $x>x_{0}$, then the ratios (23) all tend to zero as $x$ tends to infinity, and so some of the ratios of type (22) do not tend to zero. Hence

$$
\frac{x^{M_{0}} y(x)^{M_{1}} \cdots y(x+m-1)^{M_{m}}}{y(x+m)}>\frac{1}{N}>0 .
$$


That is,

$$
y(x+m)<N x^{M_{0}} y(x)^{M_{1}} \cdots y(x+m-1)^{M_{m}} \quad \text { for } x \geqq x_{0} .
$$

Hence

$$
\begin{aligned}
& \lim _{p \rightarrow \infty} \frac{y\left(x_{0}+p+m\right)}{e_{2}\left(A\left(x_{0}+p+m\right)\right)} \\
& \quad \leqq \lim _{p \rightarrow \infty} \frac{\left\{\left(x_{0}+p\right) N \cdots y\left(x_{0}+m-1\right)\right\}^{(K+1)^{p+2}}}{e_{2}\left(A\left(x_{0}+p+m\right)\right)}=0 .
\end{aligned}
$$

But this is contrary to the hypothesis that $y(x) \geqq e_{2}(A x)$ for all $x>x_{0}$, and hence $y(x)$ does not exceed $e_{2}(A x)$ for all $x>x_{0}$.

If $y(x) \leqq-e_{2}(A x)$ for all $x>x_{0}$, then we take $y(x)=-Y(x)$ and argue as above. Since $y(x)$ is continuous the theorem follows.

9. Proof of Theorem 5. The ratios $T / T^{\prime}$ take the forms

$$
\begin{gathered}
b\left\{\frac{x^{K_{0}} y(x)^{K_{1}} \cdots y(x+m-1)^{K_{m}}}{y(x+m)}\right\}^{\beta_{m}^{\prime}-\beta_{m}}, \quad \beta_{m}^{\prime}>\beta_{m}, \\
b\left\{\frac{x^{p_{0}} y(x)^{p_{1}} \cdots y(x+m-2)^{p_{m-1}}}{y(x+m-1)}\right\}^{\beta_{m-1}^{\prime}-\beta_{m-1}}, \beta_{m-1}^{\prime}>\beta_{m-1}, \\
b\left\{\frac{x^{k_{0}}}{y(x)^{k_{1}} \cdots y(x+m-2)^{k_{m-1}}}\right\},
\end{gathered}
$$

respectively, where $k_{1}, k_{2}, \cdots$ are all non-negative constants and $k_{0}$ is negative if all $k_{1}, k_{2}, \cdots, k_{m-1}$ are zero. Let

$$
\begin{aligned}
& \left|K_{1}\right|+\left|K_{2}\right|+\cdots+\left|K_{m}\right|=A_{m}, \\
& \left|p_{1}\right|+\left|p_{2}\right|+\cdots+\left|p_{m-1}\right|=B_{m},
\end{aligned}
$$

and let $\alpha_{1}$ be $\max A_{m}$ for all the ratios of type (24) and let $\alpha_{2}$ be $\max B_{m}$ for all the ratios of type (25) and let ${ }^{6}$

$$
A=1+2 \max \left(1, \log \alpha_{1}, \log \alpha_{2}\right) \text {. }
$$

We may suppose that $y(x)$ is nondecreasing for $x>x_{0}$. If $y(x)$ $\geqq e_{2}(A x)$ for a sequence of values of $x$ tending to infinity, then by Lemma 3

$y(x+1) \geqq \exp (x), \quad y(x+1) \geqq(y(x))^{B}, \quad y(x+2) \geqq(y(x+1))^{B}$, where $0<B<\exp (A / 2)$, for $x=x_{1}, x_{2}, \cdots, x_{n} \rightarrow \infty$. By hypothesis (6) all the ratios of type (26) approach zero as $x$ tends to infinity. Further for $x>x_{0}(\epsilon)$ 


$$
\begin{aligned}
& x^{K_{0}} y(x)^{K_{1}} \cdots y(x+m-1)^{K_{m}}<y(x+m-1)^{\alpha_{1}+\epsilon}, \\
& x^{p_{0}} y(x)^{p_{1}} \cdots y(x+m-2)^{p_{m-1}}<y(x+m-2)^{\alpha_{2}+\epsilon}
\end{aligned}
$$

so all the ratios of types (24) and (25) tend to zero as $x=x_{n}+2-m$ tends to infinity. Hence we have a contradiction and so

$$
y(x)<e_{2}(A x) \quad \text { for } x>x_{0} .
$$

Since $y(x)$ is nondecreasing

$$
|y(x)|<e_{2}(A x) \quad \text { for } x>x_{0} .
$$

The corollary follows immediately from the theorem. The proof of Theorem 6 is similar to the proofs of the preceding theorems.

10. Proof of Theorem 7. The ratios $T / T^{\prime}$ take the forms

$$
b\left\{\frac{x^{k_{00}} y(x)^{k_{01}} \cdots y(x+m-1)^{k_{0}, m}}{y(x+m)}\right\}^{\beta_{m}^{\prime}-\beta_{m}} \quad\left(\beta_{m}^{\prime}>\beta_{m}\right),
$$

$(27 . r+1) \quad b\left\{\frac{x^{k_{r 0}} \cdots y(x+m-r-1)^{k_{r, m-r}}}{y(x+m-r)}\right\}^{\beta_{m-r-\beta_{m-r}}^{\prime}}$

$$
\left(\beta_{m \rightarrow r}^{\prime}>\beta_{m-r}\right),
$$

$(27 . m+1)$

$$
b\left\{\frac{x^{k_{m 0}}}{y(x)}\right\}^{\beta_{0}^{\prime}-\beta_{0}}
$$

Let $m(r, s)$ be the maximum value of $\left|k_{r s}\right|$ for all the ratios of type $(27 . r+1)$ and let

$$
A_{2}=2+\max m(r, s) \quad(0 \leqq r, 0 \leqq s, r+s \leqq m) .
$$

Suppose if possible

$$
\lim _{x \rightarrow \infty} \log |y(x)| / \log x \geqq A_{2}
$$

then

$$
|y(x)| \geqq x^{A_{2}-\boldsymbol{\theta}} \quad \text { for all } x>x_{0}(\epsilon) \text {. }
$$

Let $x=x_{n}=a+n$. Then

$$
\left|\frac{y(x+r-1)^{1+k_{0} r}}{y(x+r)}\right|<c_{1}=\text { constant }
$$


for $x=x_{n}$. Further

$$
\left\{x^{k 00} / y(x)\right\}
$$

tends to zero as $x=x_{n} \rightarrow \infty$. Hence all the ratios $T / T^{\prime}$ of type (27.1) tend to zero as $x=x_{n} \rightarrow \infty$. Similarly all the ratios of types (27.2) . . $(27 . m+2)$ tend to zero as $x=x_{n} \rightarrow \infty$. Hence we have a contradiction and so the theorem is proved.

Remark. If $y(x)$ satisfies the conditions

$$
|y(x+1)| \geqq c|y(x)|^{A_{2}} \quad \text { for all } x>x_{0},
$$

$A_{2}$ being the constant defined in the proof of Theorem 7, and

$$
|y(x)| \rightarrow \infty
$$

as $x \rightarrow \infty$,

we would get, as in Theorem 7, that

$$
\lim \sup \log |y(x)| / \log x<A_{2} \text {. }
$$

But from (28) and (29) we have

$$
\liminf _{x \rightarrow \infty} \log \log |y(x)| / x \geqq \log A_{2}
$$

which contradicts (30). Hence if $y(x)$ be a solution of an algebraic difference equation of the $m$ th order, $y(x)$ cannot satisfy both the conditions (28) and (30).

MUSLIM UNIVERSITY 